\title{
High burden of private mutations due to explosive human population growth and purifying selection
}

Feng Gao, Alon Keinan*

From SNP-SIG 2013: Identification and annotation of genetic variants in the context of structure, function, and disease

Berlin, Germany. 19 July 2013

\begin{abstract}
Background: Recent studies have shown that human populations have experienced a complex demographic history, including a recent epoch of rapid population growth that led to an excess in the proportion of rare genetic variants in humans today. This excess can impact the burden of private mutations for each individual, defined here as the proportion of heterozygous variants in each newly sequenced individual that are novel compared to another large sample of sequenced individuals.
\end{abstract}

Results: We calculated the burden of private mutations predicted by different demographic models, and compared with empirical estimates based on data from the NHLBI Exome Sequencing Project and data from the Neutral Regions (NR) dataset. We observed a significant excess in the proportion of private mutations in the empirical data compared with models of demographic history without a recent epoch of population growth. Incorporating recent growth into the model provides a much improved fit to empirical observations. This phenomenon becomes more marked for larger sample sizes, e.g. extrapolating to a scenario in which 10,000 individuals from the same population have been sequenced with perfect accuracy, still about 1 in 400 heterozygous sites (or about 6,000 variants) at the 10,001 $1^{\text {st }}$ individual are predicted to be novel, 18-times as predicted in the absence of recent population growth. The proportion of private mutations is additionally increased by purifying selection, which differentially affect mutations of different functional annotations.

Conclusions: The burden of private mutations for each individual, which are singletons (i.e. appearing in a single copy) in a larger sample that includes this individual, is predicted to be greatly increased by recent population growth, as well as by purifying selection. Comparison with empirical data supports that European populations have experienced recent rapid population growth, consistent with previous studies. These results have important implications to the design and analysis of sequencing-based association studies of complex human disease as they pertain to private and very rare variants. They also imply that personalized genomics will indeed have to be very personal in accounting for the large number of private mutations.

\section{Background}

Many recent studies that sequenced large numbers of individuals have shown that human populations have experienced a complex demographic history, including a recent epoch of rapid growth in effective population size, although estimates have varied greatly among studies [1-7]. The growth of European population has recently

\footnotetext{
* Correspondence: ak735@cornell.edu

Department of Biological Statistics and Computational Biology, Cornell University, Ithaca, NY 14853, USA
}

been estimated to be exponential with a rate of $2-5 \%$ pergeneration increase in population size [1,3,7]. This recent growth has resulted in an excess of rare single nucleotide variants (SNVs), commonly defined as those with a minor allele (the less common of the two alleles) frequency (MAF) of less than $0.5 \%$ (or 1\%) in a sample of individuals from the same population [e.g. [5,8]]. The proportion of singletons (SNVs with only one copy in the entire sample) is especially elevated due to this recent rapid growth $[1,3,5,7,9]$. Consequently, the corresponding site frequency 
spectrum (SFS), a summary statistic that indicates the proportion of variants of each possible allele count in the sample (e.g. Figure 1) is skewed towards lower allele counts.

A predicted consequence of the skew in the SFS due to population growth is an increase in the burden of private mutations for each individual. We recently defined this quantity as the proportion of heterozygous positions in each newly sequenced individual that are novel, i.e., completely absent from a previously sequenced sample from the same population [9]. In that previous paper, we observed this burden to be higher in samples from populations of European and East Asian descent than is predicted by previously estimated demographic models that do not include an epoch of recent population growth [9]. However, empirical estimates in that paper were based on a small sample size of less than 100 individuals, while the contribution of recent rapid growth is expected to be more pronounced for larger sample sizes [1-6,9].

Here, we set out to (1) empirically estimate the burden of private mutations from large samples of individuals of European ancestry, (2) compare these estimates with predictions of previously proposed demographic models with and without a recent epoch of exponential growth $[3,10]$, and (3) contrast SNVs of different functions that are expected to have undergone different selective effects. As purifying, negative selection on deleterious SNVs skews the SFS towards rare variants [1,5,11-13], it can interact with the effect of recent population growth in increasing the burden of private SNVs, and differently so for different functional categories. With the rapidly decreasing cost of sequencing, more and more high-quality sequencing data sets of large sample sizes and improved accuracy of detecting rare variants become available. This provides an excellent opportunity for a more accurate study of the burden of private mutations. In this paper, we considered two such sequencing data sets of samples from populations of European ancestry: the NHLBI Exome Sequencing Project (ESP) [1][7] and the Neutral Regions (NR) data set of putatively neutral regions [3].

\section{Results and discussion}

In all analyses, we contrast three different demographic models and the fit of their predictions to the NR data set [3] and to 7 functional categories of the ESP data set $[1,7]$. The three demographic models are (1) a population that has been of constant population size throughout history, (2) a model of European history that includes two population bottlenecks [10], and (3) a model of European history with two bottlenecks, a recent change in population size, followed by a recent epoch of rapid population growth [3] (Model II therein).

\section{Comparison of site frequency spectra}

As the burden of private mutations is a function of the site frequency spectrum, we first contrasted the site frequency spectra between three demographic models, the NR data [3], and the ESP data [1,7] (Figure 1). In order to allow comparison of the data sets with different sample sizes, as well as account for missing genotype calls for

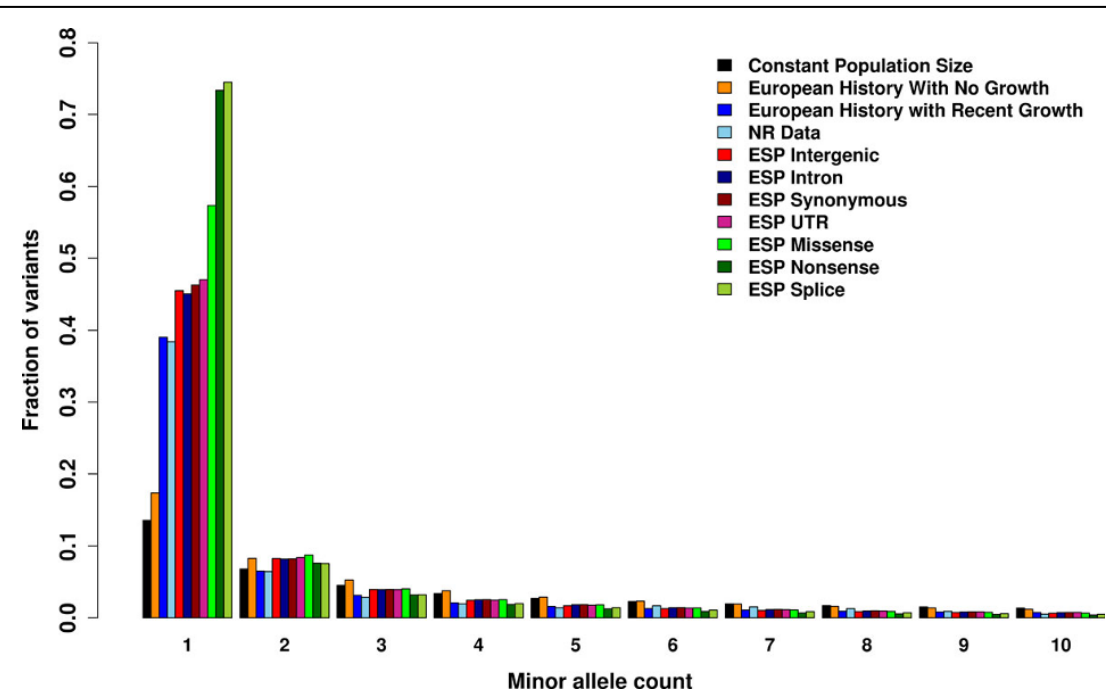

Figure 1 Site frequency spectra of demographic models and data with a sample size of $\mathbf{9 0 0}$. The SFS for 3 demographic models, the Neutral Regions (NR) data and 7 categories of the Exome Sequencing Project (ESP) data. To adjust for the different sample sizes in the two datasets, probabilistic subsampling was applied to make all sample sizes equal to 900 chromosomes. Only the first 10 minor allele count categories are shown. For each minor allele count, from left to right: constant population size, European history with 2 bottlenecks but no growth [10], European history with recent growth (Model II in [3]), the NR data, intergenic SNVs of the ESP data, intron SNVs of the ESP data, synonymous SNVs of the ESP data, UTR SNVs of the ESP data, missense SNVs of the ESP data, nonsense SNVs of the ESP data and splice SNVs of the ESP data. 
each SNV, we probabilistically subsampled all data to a sample size of 900 haploid chromosomes (Methods).

The proportion of singletons from demographic models (1) and (2) is greatly lower than that in the observed data and that predicted by model (3), where recent growth is incorporated (Figure 1). Among the categories of the ESP data, categories that are expected to be more functional show a higher proportion of singletons, e.g. intronic, intergenic, synonymous, and UTR SNVs have a significantly lower proportion than non-synonymous, nonsense, and splice SNVs (Figure 1), which is expected by the latter being more often deleterious. These results recapitulate those from the ESP [7]. The proportion of singletons in the SNVs from the NR data is lower than all categories of SNVs from ESP, which is consistent with the former being designed such that variants are very far from genes and putatively neutral [3], while the latter consists of variants in and near protein-coding genes [1,7], which are expected to more often be targeted by purifying selection. Another factor that can contribute to this difference between the NR and ESP datasets is that the former aimed to capture a sample of homogenous ancestry, which corresponds to NorthWestern European ancestry [3], while the latter consists of a broad sample of European Americans that exhibits a higher level of population structure $[1,7]$. Increased population structure can lead to an increase in the proportion of rare variants since some of these can be due to mutations that postdate the split of the population captured by the different ancestries [3].

\section{Comparison of the burden of private mutations}

The predicted burden of private mutations for each individual from all demographic models and the empirical burden observed in the different data sets and functional categories are presented in Figure 2. Across all sample sizes, the burden of private mutations from empirical data is significantly higher than that predicted by demographic models without growth. For example, based on the results of the NR data, when 100 individuals have been sequenced, we estimated that about $1.4 \%$ out of all heterozygous sites in the $101^{\text {st }}$ sequenced individual are novel, that is specific to the $101^{\text {st }}$ individual and completely absent from the first set of 100 individuals. While models

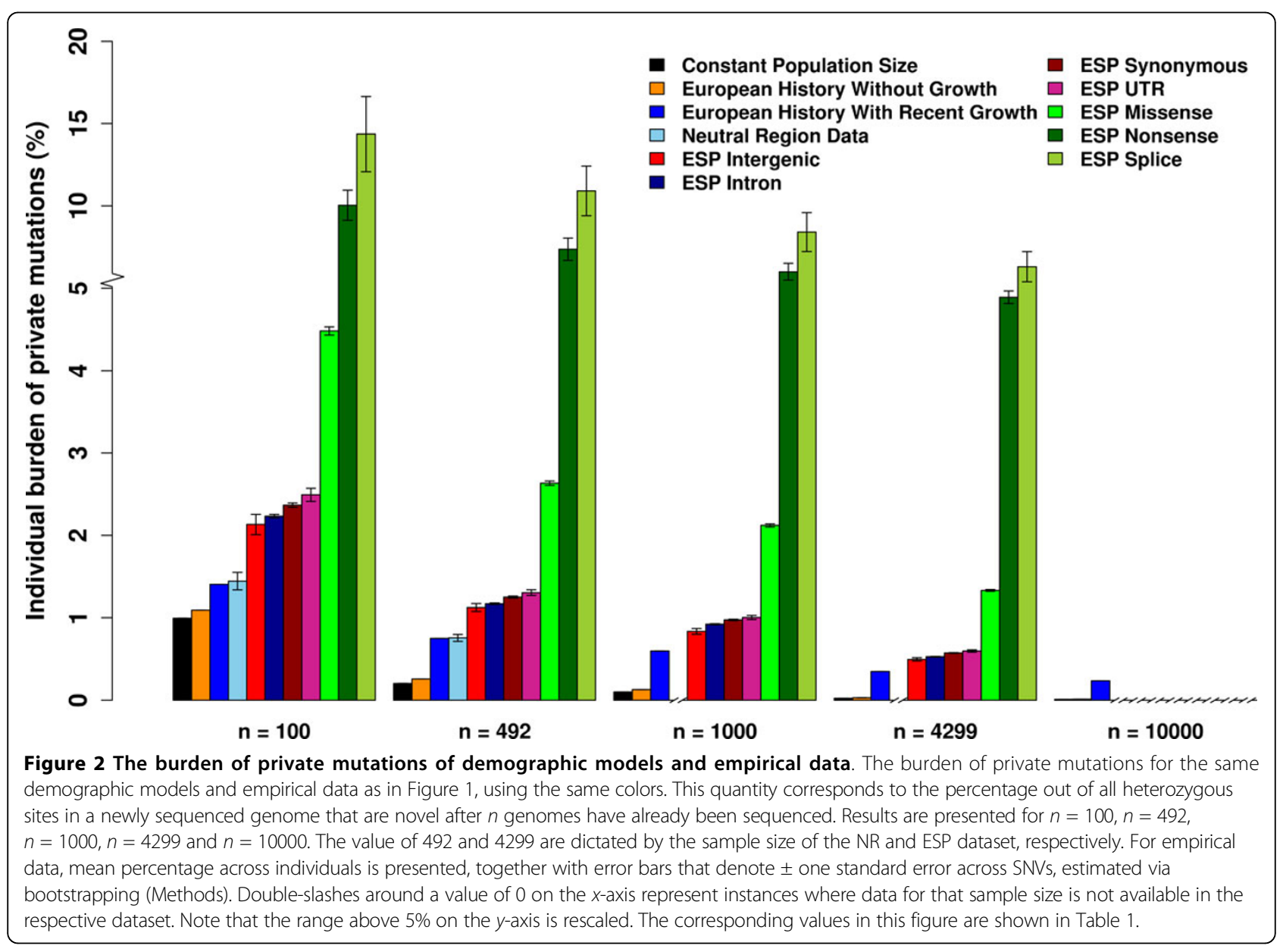


(1) and (2) predict only $1 \%$ in this scenario, model (3) is consistent with this estimate in the NR data.

For all demographic models and observed data, as more individuals are sequenced, the burden of private mutations decreases (Figure 2), because increasing sample size makes it more probable that a variant has already been discovered [9]. At the same time, the effect of recent growth itself on the burden of private mutations is much more pronounced with increasing sample size. For example, for the NR data, when 492 individuals are sequenced, the estimated burden of mutation from the $493^{\text {rd }}$ sequenced individual is about $0.76 \%$ (Table 1 ). The estimations from models (1) and (2) are only $0.20 \%$ and $0.26 \%$, respectively, about a third of empirical data, while model (3) matches the data well. We note that this percentage varies greatly across individuals with the relatively small number of SNVs in the NR data (Table 2).

When extrapolating the models to consider a scenario in which 10,000 individuals are sequenced, model (3) predicts the burden of mutations of the $10,001^{\text {st }}$ individual to be $0.24 \%$ (Table 1), 24-times and 18-times that from models without recent growth that predict $0.010 \%$ and $0.013 \%$ based on models (1) and (2), respectively (Table 1). This corresponds to almost 1 of 400 heterozygous positions, which is equivalent to about 6,000 variants genome-wide. This estimate is at least two orders of magnitude larger than the expected number of de novo mutations of each individual [e.g. [14]]. Hence, we predict that thousands of novel variants will be discovered in each newly sequenced genome even after tens of thousands of genomes from exactly the same population have already been sequenced with perfect accuracy, and that these are rarely due to de novo mutations.

Another important observation is that the burden of private mutations for each individual calculated from all seven categories of the ESP data is consistently higher than that from the NR data for all sample sizes (Figure 2). This is consistent with the observation that the SFS of the ESP data are more left-skewed than those of the NR data, which is consistent with decreased effect of purifying selection and population structure on the latter. Comparing the different ESP categories, splice and non-sense SNVs, which are expected to most often be deleterious, have the largest burden of private mutations across all sample sizes. Similarly, the burden of all functional categories is ordered by common expectations as to how often such mutations are expected to be functional. The burden of private mutations captures a unique summary of the SFS that more clearly shows the effect of purifying selection. For example, when $n=492$, the proportion of singletons is $46.2 \%$ for the ESP intergenic SNVs and $74.8 \%$ for the ESP splice SNVs, which is 1.6-fold. In comparison, the burden of private mutations for splice SNVs is about 9.7-fold of that for intergenic SNVs. This difference is even more pronounced when the sample size is larger, with 12.7-fold different when $n=4299$ (Figure 2).

\section{Conclusions}

Recent whole-genome sequencing data sets show that the proportion of rare variants in large samples, especially

Table 1 Estimated mean and standard error of percentage of private mutations for each individual.

\begin{tabular}{|c|c|c|c|c|c|}
\hline Group & $n=100$ & $n=492$ & $n=1000$ & $n=4299$ & $n=10000$ \\
\hline $\begin{array}{l}\text { Constant Population } \\
\text { Size Model }\end{array}$ & $0.995 \%$ & $0.203 \%$ & $0.100 \%$ & $0.023 \%$ & $0.010 \%$ \\
\hline European History with Two Bottlenecks & $1.092 \%$ & $0.257 \%$ & $0.129 \%$ & $0.031 \%$ & $0.013 \%$ \\
\hline European History with Recent Growth & $1.406 \%$ & $0.750 \%$ & $0.596 \%$ & $0.349 \%$ & $0.237 \%$ \\
\hline NR Data & $\begin{array}{c}1.444 \% \\
(0.106 \%)\end{array}$ & $\begin{array}{l}0.756 \% \\
(0.043 \%)\end{array}$ & NA & NA & NA \\
\hline ESP Intergenic & $2.132 \%(0.123 \%)$ & $\begin{array}{l}1.125 \% \\
(0.049 \%)\end{array}$ & $\begin{array}{c}0.835 \% \\
(0.034 \%)\end{array}$ & $0.496 \%(0.019 \%)$ & NA \\
\hline ESP Intron & $2.233 \%(0.022 \%)$ & $\begin{array}{l}1.171 \% \\
(0.009 \%)\end{array}$ & $\begin{array}{l}0.922 \% \\
(0.007 \%)\end{array}$ & $\begin{array}{c}0.528 \% \\
(0.004 \%)\end{array}$ & NA \\
\hline ESP Synonymous & $\begin{array}{l}2.366 \% \\
(0.026 \%)\end{array}$ & $\begin{array}{l}1.252 \% \\
(0.012 \%)\end{array}$ & $\begin{array}{l}0.974 \% \\
(0.009 \%)\end{array}$ & $\begin{array}{l}0.573 \% \\
(0.005 \%)\end{array}$ & NA \\
\hline ESP UTR & $\begin{array}{l}2.492 \% \\
(0.079 \%)\end{array}$ & $\begin{array}{l}1.305 \% \\
(0.034 \%)\end{array}$ & $\begin{array}{l}1.004 \% \\
(0.025 \%)\end{array}$ & $\begin{array}{l}0.596 \% \\
(0.014 \%)\end{array}$ & NA \\
\hline ESP Missense & $\begin{array}{l}4.482 \% \\
(0.049 \%)\end{array}$ & $\begin{array}{l}2.632 \% \\
(0.026 \%)\end{array}$ & $\begin{array}{l}2.121 \% \\
(0.019 \%)\end{array}$ & $\begin{array}{l}1.333 \% \\
(0.011 \%)\end{array}$ & NA \\
\hline ESP Nonsense & $\begin{array}{l}10.04 \% \\
(0.92 \%)\end{array}$ & $\begin{array}{l}7.37 \% \\
(0.68 \%)\end{array}$ & $\begin{array}{l}6.00 \% \\
(0.50 \%)\end{array}$ & $\begin{array}{l}4.46 \% \\
(0.38 \%)\end{array}$ & NA \\
\hline ESP Splice & $\begin{array}{l}14.36 \% \\
(2.29 \%)\end{array}$ & $\begin{array}{l}10.91 \% \\
(1.50 \%)\end{array}$ & $\begin{array}{l}8.41 \% \\
(1.19 \%)\end{array}$ & $\begin{array}{l}6.31 \% \\
(0.91 \%)\end{array}$ & NA \\
\hline
\end{tabular}

The burden of private mutations for $n=100, n=492, n=1000, n=4299$ and $n=10000$, the corresponding values for Figure 2 and shown here for completeness. The number in parenthesis denotes the standard error across SNVs estimated via bootstrap (Methods). NA indicates that the data for that sample size is not available in the respective dataset. 
Table 2 The mean and standard deviation of the burden of private mutations across individuals.

\begin{tabular}{cc}
\hline Group & $\begin{array}{c}\text { The Burden of Private } \\
\text { Mutations }\end{array}$ \\
\hline $\begin{array}{c}\text { Constant Population Size Model } \\
\text { European History with Two } \\
\text { Bottlenecks }\end{array}$ & $0.208 \%(0.299 \%)$ \\
European History with Recent & $0.276 \%(0.352 \%)$ \\
Growth & $0.736 \%(0.614 \%)$ \\
NR Data & $0.758 \%(0.852 \%)$ \\
\hline
\end{tabular}

The burden of private mutations and the standard deviation of the sample for three demographic models and the NR data. The results correspond to $n=$ 492, the sample size of the NR data less one, as they are based on the individuals from that dataset. These results are not based on randomized chromosomes, but rather on the actual genotype information for each individual in turn. For the three demographic models, sequences were simulated with the same number of SNVs as in the NR data (Methods). The number in parenthesis denotes the standard deviation of the sample. These large standard deviations suggest a significant variation in percentage of private mutations across individuals when the small number of SNVs from the NR dataset is considered.

singletons, is significantly elevated compared with the prediction from the standard coalescent theory that assumes a constant population size and from previous demographic models without recent growth $[1,3,7,9]$. Recent demographic modeling studies predict that humans have experienced a recent and rapid population growth, which explains an increased proportion of singletons and other rare variants [1-6]. In this paper, we examined the burden of private mutations for each individual, a statistic that reflects the relationship between the relative proportions of singletons and more common variants contained in a sample, with three demographic models and two data sets under different sample sizes. We found that the burden of private mutations calculated from empirical data and estimated from demographic models with a recent growth is significantly higher than that estimated from models without recent growth across all sample sizes. The discrepancy is predicted to be much more pronounced for larger number of sequenced individuals. We showed that this finding is consistent with a recent epoch of population growth. Moreover, we found that the SNVs that are affected by stronger purifying selection will generally have larger burden of private mutations compared with more selectively neutral SNVs, since they will have a higher proportion of singletons.

The proportion of private mutations that we consider translates to the number of novel variants expected to be ascertained with each newly sequenced genome. Hence, our results have implications to sequencingbased association studies of complex human diseases and other sequencing studies. For instance, we predict that even after 10,000 individuals from the exact same European population have been perfectly sequenced, still 1 in 400 heterozygous sites will be novel in each newly sequenced genome, which corresponds to discovering about 6,000 new variants. This large expectation is due to the effect of the recent rapid growth of European populations, which leads to this number being at least 18 -fold that predicted in the absence of such growth. Hence, careful consideration must be given to private mutations in the design and analysis of sequencing-based association studies and in quantifying the role played by rare variants in complex human disease [15-19].

\section{Methods Datasets}

Two data sets were used in this study. The NR data contains the genotypes of 493 European individuals with high homogeneity on relatively neutral SNVs of 15 genetic regions [3]. For quality purposes, all SNVs with less than 900 successful genotype counts were filtered from the analysis. The remaining 1,746 SNVs constitute $95 \%$ of all variants [3]. The summarized data of 4,300 European individuals from NHLBI Exome Sequencing Project records the minor allele count and major allele count of each SNV identified in 15,585 genes on all chromosomes (including chromosome $x$ and $Y$ ) $[1,7]$. In this analysis, we combined all of the autosomal SNVs according to the 7 categories: intergenic, intron, missense, nonsense, splice, synonymous and UTR. For quality purpose, SNVs are filtered if the average read depth is less than or equal to 20 or the successful genotype counts are less than 8,170 (95\%).

\section{Subsampling approach}

In order to compare the SFS of data with different sample sizes (including the different sample sizes across the SNVs caused by unsuccessful genotype counts in the same data set), all the observed data were subsampled to 900 chromosomes. Following the strategy used in [10], for a SNV with $j$ minor alleles out of $n$ successful genotype counts, the probability that it is of $x$ minor alleles when subsampled to $m$ chromosomes is

$$
P(x \text { of } m)=\frac{1}{\delta(x, m-x)}\left(\frac{\left(\begin{array}{c}
j \\
x
\end{array}\right)\left(\begin{array}{c}
n-j \\
m-x
\end{array}\right)}{\left(\begin{array}{c}
n \\
m
\end{array}\right)}+\frac{\left(\begin{array}{c}
j \\
m-x
\end{array}\right)\left(\begin{array}{c}
n-j \\
x
\end{array}\right)}{\left(\begin{array}{c}
n \\
m
\end{array}\right)}\right)
$$

where $\delta(a, b)=1$ if $a=b$ and $\delta(a, b)=0$ if $a \neq b$, $x=0,1,2, \ldots,\left[\frac{m}{2}\right]$ and $\left(\begin{array}{l}a \\ b\end{array}\right):=0$ if $a<b$.

\section{Expected SFS and the burden of private mutations for demographic models}

The SFS of the three demographic models were calculated using exact computation [20] instead of simulations.

For a demographic model with constant population size, the burden of private mutations can be derived 
under standard coalescent theory [21]. For constant population size, the expected number of singletons of a folded SFS for a sample of $(n+1)$ diploid individuals is

$$
E\left[\eta_{1}\right]=\theta\left(1+\frac{1}{2 n+1}\right)
$$

where $\theta=4 N \mu$. The expected number of singletons that belong to one individual is

$$
E[s]=\frac{1}{n+1} E\left[\eta_{1}\right]=\frac{2 \theta}{2 n+1}
$$

The expected number of heterozygote sites for the pair of sequences from one individual $E[h]=\theta$. Thus the expected burden of private mutations is

$$
E[\alpha]=\frac{E[s]}{E[h]}=\frac{2}{2 n+1}
$$

For variable population size, the general solution is

$$
E[\alpha]=\frac{1}{n+1} \frac{E\left[T_{2 n+2,1}\right]+E\left[T_{2 n+2,2 n+1}\right]}{E\left[T_{2,1}\right]}
$$

where $T_{p, q}$ stands for the total length of all branches in the coalescent tree which have exactly $q$ descents out of the total number of descents $p$. The branch lengths are calculated by exact computation [20].

\section{Computation of the burden of private mutations using data sets and simulations}

For the NR data, for each of the 493 individuals, the burden of private mutations $\alpha$ is directly calculated by the proportion of heterozygote sites which contain singletons using the individual genotypes. Missing genotypes were abandoned. The mean and standard deviation of $\alpha$ for this sample were then calculated by

$$
\bar{\alpha}=\frac{1}{n} \sum_{i=1}^{n} \alpha_{i}, s(\alpha)=\sqrt{\frac{\sum_{i=1}^{n}\left(\alpha_{i}-\bar{\alpha}\right)^{2}}{n-1}}
$$

where $n$ is the sample size and equals 493 here.

For ESP data and demographic models, as the individual genotypes were not available, sequences were simulated by distributing the minor alleles of each SNV to individuals randomly and independently. Unsuccessful genotype calls (missing genotypes) were also distributed randomly to the individuals but were distributed in pairs. In other words, the genotypes of each individual at each site either were both existent or both missing. Then $\alpha$ was calculated using these simulated sequences in the same way as for the NR data.

For the demographic histories from which we can only get the SFS, a similar method is applied. Namely we simulated a certain number of SNVs according to the
SFS and randomly assigned the minor alleles into individual sequences. The simulated sequences were paired randomly to form the sequences of an individual and $\alpha$ for each individual was then calculated.

To calculate $\alpha$ for a smaller sample size $m, m$ individuals were randomly chosen from the original $n$ individuals and $\alpha$ was calculated using the genotypes from these $m$ individuals with the previously stated approach.

To study the effects of limited sites, a bootstrap approach was applied. Specifically, we resampled individual SNPs with replacement for 1,000 times. For each bootstrap, we calculated the average $\alpha\left(\alpha_{\mathrm{b}, i}\right)$ across all individuals and these 1,000 averages were used to calculate the mean and standard deviation of the bootstrap, the latter of which is an estimate of the standard error of the sample:

$$
\bar{\alpha}_{\mathrm{b}}=\frac{1}{n_{\mathrm{b}}} \sum_{i=1}^{n_{\mathrm{b}}} \alpha_{\mathrm{b}, i}, s_{\mathrm{b}}(\alpha)=\sqrt{\frac{\sum_{i=1}^{n_{\mathrm{b}}}\left(\alpha_{\mathrm{b}, i}-\bar{\alpha}_{\mathrm{b}}\right)^{2}}{n_{\mathrm{b}}-1}}
$$

where $n_{\mathrm{b}}$ is the number of bootstraps and equals 1,000 here.

\section{Competing interests}

The authors declare that they have no competing interests.

\section{Authors' contributions}

FG carried out all analyses. AK conceived the experiments and provided materials. Both authors co-wrote the manuscript and approved its final version.

\section{Acknowledgements}

We thank Diana Chang for helpful comments on previous versions of this manuscript. This work was supported in part by National Institutes of Health Grant R01HG006849. A.K. was also supported by The Ellison Medical Foundation and the Edward Mallinckrodt, Jr. Foundation.

\section{Declarations}

The publication costs for this article were funded by the above grant. This article has been published as part of BMC Genomics Volume 15 Supplement 4, 2014: SNP-SIG 2013: Identification and annotation of genetic variants in the context of structure, function, and disease. The full contents of the supplement are available online at http://www.biomedcentral.com/ bmcgenomics/supplements/15/S4

Published: 20 May 2014

\section{References}

1. Tennessen JA, Bigham AW, O'Connor TD, Fu W, Kenny EE, Gravel S, McGee S, Do R, Liu X, Jun G, Kang HM, Jordan D, Leal SM, Gabriel S, Rieder MJ, Abecasis G, Altshuler D, Nickerson DA, Boerwinkle E, Sunyaev S, Bustamante CD, Bamshad MJ, Akey JM, Broad GO, Seattle GO: Evolution and functional impact of rare coding variation from deep sequencing of human exomes. In Science. Volume 337. NHLBI Exome Sequencing Project; 2012:(6090):64-69.

2. Gravel S, Henn BM, Gutenkunst RN, Indap AR, Marth GT, Clark AG, Yu F, Gibbs RA: Demographic history and rare allele sharing among human populations. In Proc Natl Acad Sci USA. Volume 108. 1000 Genomes Project Bustamante CD; 2011:(29):11983-11988.

3. Gazave E, Ma L, Chang D, Coventry A, Gao F, Muzny D, Boerwinkle E, Gibbs $R$, Sing CF, Clark AG, Keinan A: Neutral genomic regions refine models of recent rapid human population growth. Proc Natl Acad Sci USA 2014, 111(2):757-762

4. Gutenkunst RN, Hernandez RD, Williamson SH, Bustamante CD: Inferring the joint demographic history of multiple populations from multidimensional SNP frequency data. PLOS Genet 2009, 5(10):e1000695. 
5. Nelson MR, Wegmann D, Ehm MG, Kessner D, St Jean P, Verzilli C, Shen J, Tang Z, Bacanu SA, Fraser D, Warren L, Aponte J, Zawistowski M, Liu X, Zhang H, Zhang Y, Li J, Li Y, Li L, Woollard P, Topp S, Hall MD, Nangle K, Wang J, Abecasis G, Cardon LR, Zöllner S, Whittaker JC, Chissoe SL, Novembre J, Mooser V: An abundance of rare functional variants in 202 drug target genes sequenced in 14,002 people. Science 2012, 337(6090):100-104.

6. Coventry A, Bull-Otterson LM, Liu X, Clark AG, Maxwell TJ, Crosby J, Hixson JE, Rea TJ, Muzny DM, Lewis LR, Wheeler DA, Sabo A, Lusk C, Weiss KG, Akbar H, Cree A, Hawes AC, Newsham I, Varghese RT, Villasana D, Gross S, Joshi V, Santibanez J, Morgan M, Chang K, IV WH, Templeton AR,

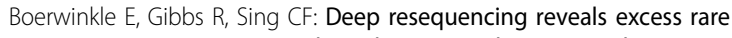
recent variants consistent with explosive population growth. Nat Commun 2010, 1:131.

7. Fu W, O'Connor TD, Jun G, Kang HM, Abecasis G, Leal SM, Gabriel S, Rieder MJ, Altshuler D, Shendure J, Nickerson DA, Bamshad MJ, NHLBI Exome Sequencing Project, Akey JM: Analysis of 6,515 exomes reveals the recent origin of most human protein-coding variants. Nature 2013, 493(7431):216-220.

8. Gibson G: Rare and common variants: twenty arguments. Nat Rev Genet 2012, 13(2):135-145.

9. Keinan A, Clark AG: Recent explosive human population growth has resulted in an excess of rare genetic variants. Science 2012, 336(6082):740-743

10. Keinan A, Mullikin JC, Patterson N, Reich D: Measurement of the human allele frequency spectrum demonstrates greater genetic drift in East Asians than in Europeans. Nat Genet 2007, 39(10):1251-1255.

11. Charlesworth B, Morgan MT, Charlesworth D: The effect of deleterious mutations on neutral molecular variation. Genetics 1993, 134(4):1289-1303.

12. Kiezun A, Garimella K, Do R, Stitziel NO, Neale BM, McLaren PJ, Gupta N, Sklar P, Sullivan PF, Moran JL, Hultman CM, Lichtenstein P, Magnusson P, Lehner T, Shugart YY, Price AL, de Bakker PI, Purcell SM, Sunyaev SR: Exome sequencing and the genetic basis of complex traits. Nat Genet 2012, 44(6):623-630

13. Fay JC, Wyckoff GJ, Wu Cl: Positive and negative selection on the human genome. Genetics 2001, 158(3):1227-1234.

14. Roach JC, Glusman G, Smit AF, Huff CD, Hubley R, Shannon PT, Rowen L, Pant KP, Goodman N, Bamshad M, Shendure J, Drmanac R, Jorde LB, Hood L, Galas DJ: Analysis of genetic inheritance in a family quartet by whole-genome sequencing. Science 2010, 328(5978):636-639.

15. Manolio TA, Collins FS, Cox NJ, Goldstein DB, Hindorff LA, Hunter DJ, McCarthy MI, Ramos EM, Cardon LR, Chakravarti A, Cho JH, Guttmacher AE, Kong A, Kruglyak L, Mardis E, Rotimi CN, Slatkin M, Valle D, Whittemore AS, Boehnke M, Clark AG, Eichler EE, Gibson G, Haines JL, Mackay TF, McCarroll SA, Visscher PM: Finding the missing heritability of complex diseases. Nature 2009, 461(7265):747-753.

16. Frazer KA, Murray SS, Schork NJ, Topol EJ: Human genetic variation and its contribution to complex traits. Nat Rev Genet 2009, 10(4):241-251.

17. Maher B: Personal genomes:The case of the missing heritability. Nature 2008, 456(7218):18-21.

18. Dickson SP, Wang K, Krantz I, Hakonarson H, Goldstein DB: Rare variants create synthetic genome-wide associations. PLOS Biol 8(1):e1000294.

19. Chang D, Keinan A: Predicting signatures of "synthetic associations" and "natural associations" from empirical patterns of human genetic variation. PLoS Comput Biol 2012, 8(7):e1002600.

20. Bhaskar A, Clark AG, Song YS: Distortion of genealogical properties when the sample is very large. Proc Natl Acad Sci USA 2014, 111(6):2385-2390.

21. Marth GT, Czabarka E, Murvai J, Sherry ST: The allele frequency spectrum in genome-wide human variation data reveals signals of differential demographic history in three large world populations. Genetics 2004, 166(1):351-372.

doi:10.1186/1471-2164-15-S4-S3

Cite this article as: Gao and Keinan: High burden of private mutations due to explosive human population growth and purifying selection. BMC Genomics 2014 15(Suppl 4):S3.

\section{Submit your next manuscript to BioMed Central and take full advantage of:}

- Convenient online submission

- Thorough peer review

- No space constraints or color figure charges

- Immediate publication on acceptance

- Inclusion in PubMed, CAS, Scopus and Google Scholar

- Research which is freely available for redistribution

Submit your manuscript at www.biomedcentral.com/submit 